\title{
Cuidados no Fim da Vida: O Ensino Médico no Brasil
}

\section{PALAVRAS-CHAVE: \\ - Assistência Terminal; \\ - Cuidados Paliativos, \\ - Educação Médica; \\ - Escolas Médicas.}

\section{End-of-life Care Education in Brazilian Medical Schools}

Andréia Padilha de Toledo ${ }^{I}$ Denise Gonçalves Priolli ${ }^{I}$

\section{KEYWORDS: \\ - Terminal Care; \\ - Palliative Care; \\ - Education, Medical; \\ - Schools, Medical.}

Reencaminhado em: 27/09/2011

Reencaminhado em: 02/01/2012

Aprovado em: 06/02/2012

REVISTA BRASILEIRA DE EDUCAÇ̃̃o MÉDICA
"Trabalho desenvolvido pelo curso de Medicina da Universidade São Francisco, Bragança Paulista, São Paulo, Brasil.

${ }^{I}$ Universidade São Francisco, Bragança Paulista, SP, Brasil.

Introdução: Há importância na análise do ensino-aprendizagem na graduação médica que permita identificar oportunidades de melhoria no ensino dos cuidados no final da vida. Objetivo: Descrever atitudes e práticas do ensino dos cuidados no fim da vida no Brasil conforme relatado pelos coordenadores de curso. Método: Questionário sobre o ensino dos cuidados no fim da vida foi aplicado em 179 coordenadores de escolas de medicina brasileiras. Resultados: Cinqüenta e oito coordenadores participaram (32,4\%). A maioria (96,6\%) considerou muito importante o ensino dos cuidados no fim da vida. Setenta e três por cento acredita que o tempo para ensinar sobre os cuidados paliativos em seus currículos é insuficiente, sendo sua prioridade insuficiente ou inexistente em 50,9\% das opiniões. O pequeno número de docentes especializados foi considerado como uma das barreiras para incorporar esse ensino no currículo da graduação. Conclusão: As atitudes e práticas quanto ao ensino dos cuidados no fim da vida nas escolas médicas sugerem limitações. Embora os atuais coordenadores acreditem em sua importância, ainda é dada pouca prioridade ao ensino deste tema no Brasil.

Background: There is importance in analysis of tech-learning in the medical schools that allows identify opportunities of improvement in end-of-life care education. Purpose: The aim of this stu$d y$ is describe end-of-care teaching attitudes and practices in undergraduate medical curriculum in Brazil as reported by the course coordinators. Method: A questionnaire about end-of-life care was applied on 179 course coordinators of medical schools in Brazil. Results: Fifty-eight coordinators participated (32,4\%). Most of them (96,6\%) considered end-of-life care education as very important. Seventy-tree percent believes that the time to teaching about palliative care in their curriculum is insufficient. The priority given to education in palliative care is considered insufficient or inexistent in 50,9\% of cases. The small number of faculty expertise is one of barriers to incorporate end-life-care in the medical school's curriculum. Conclusion: Attitudes and practices suggest that end-life-care education has limitations in undergraduate medical Brazilian curriculum. Even so the current coordinators believe its importance still is given little priority to the education of this subject in Brazil. 


\section{INTRODUÇÃO}

O moderno movimento Hospice, formalmente surgido em 1967, fruto do trabalho da doutora Dame Cicely Mary Saunders com a criação do St. Christopher's Hospice, em Londres, busca agregar o ensino e a pesquisa à assistência aos pacientes com doença terminal, tornando-se marco histórico no ensino dos cuidados ao fim da vida ${ }^{1}$. Com seu início no Reino Unido, atualmente os serviços em cuidados paliativos estão presentes, em diferentes estágios de desenvolvimento, em 115 de 234 países $^{2}$. Embora exista grande avanço nas pesquisas e sua expansão esteja em progressão, a literatura demonstra que profissionais da saúde têm deficiências significativas quanto à sua capacitação ${ }^{3}$.

Em 1995 o estudo SUPPORT ${ }^{3}$, buscou avaliar indicadores de qualidade quanto ao cuidado recebido por mais de quatro mil pacientes com doenças terminais em hospitais universitários. Nesse estudo, 47\% dos médicos desconheciam o desejo dos pacientes quanto a receber manobras de ressuscitação cardiopulmonar. Trinta e oito por cento daqueles que faleceram passaram mais de dez dias em Unidades de Terapia Intensiva e estiveram, por período médio de oito dias, inconscientes, recebendo ventilação mecânica sem o conhecimento/consentimento sobre o recebimento deste cuidado. Chama mais ainda a atenção o fato do controle da dor nos últimos dias de vida, ponto de crucial importância quando se pensa em qualidade em cuidados no fim de vida ter sido precário em mais da metade dos pacientes com doença terminal.

No Brasil, não há dados definitivos sobre o ensino dos cuidados no fim da vida. A literatura em relação a este tópico ainda é bastante escassa, podendo-se inferir que exista grande deficiência sobre a capacitação em medicina paliativa entre os médicos. Este assertiva baseia-se na escassez de publicações sobre o ensino dirigido em cuidados no fim de vida em currículos de graduação das escolas médicas brasileiras ${ }^{4,5}$.

A medicina paliativa foi reconhecida recentemente como área de atuação médica no país ${ }^{6}$, tornando-se necessário que instituições de ensino e a política nacional de saúde compreendam sua importância e impulsionem sua habilitação. Neste contexto, definições quanto a atual situação e melhor estratégia de ensino-aprendizagem abordada nas escolas médicas se faz importante. Adicionalmente, com a mudança do Código de Ética Médica e, sobretudo com a aceitação da ortotanásia como elemento fundamental à manutenção da dignidade no final da vida ${ }^{7}$, torna-se relevante e necessária a discussão sobre o ensino dos cuidados no fim da vida, tema, muitas vezes negligenciado no ensino da graduação em medicina no Brasil.

\section{OBJETIVO}

O objetivo do estudo é descrever atitudes e práticas do ensino dos cuidados no fim da vida conforme relatado pelos coordenadores de curso nas escolas de Medicina do Brasil.

\section{MATERIAL E MÉTODO}

A realização desse estudo obedeceu todas as etapas previstas pelo Comitê de Ética em Pesquisa da Universidade São Francisco (CAAE 0137.0.142.000-09) e as exigências do Conselho de Ética em Pesquisa da Comissão Nacional de Ética em Pesquisa (CONEP) do Ministério da Saúde (Resolução CNS196/96). Todos os participantes, por meio do Termo de Consentimento Livre e Esclarecido (TCLE), concordaram e receberam os devidos esclarecimentos em relação aos objetivos e a metodologia a serem empregadas no estudo.

\section{Amostra}

Participaram do estudo 58 coordenadores de 179 cursos de medicina do Brasil. A lista dos coordenadores foi obtida a partir do cadastro das instituições de ensino superior do Ministério da Educação ${ }^{8}$. O contato com os respectivos coordenadores dos cursos foi obtido por meio de websites das respectivas escolas médicas e no website da Associação Brasileira de Educação Médica (ABEM $)^{9}$.

\section{Instrumento de Pesquisa e Coleta de Dados}

Trata-se de estudo descritivo transversal. Os dados foram obtidos a partir de questionário com foco nas atitudes e práticas no ensino dos cuidados no fim da vida no curriculum da graduação médica, adaptado do estudo de Sullivan et al. ${ }^{10}, 2004$. Foi feita a definição de cuidados paliativos e seus princípios, conforme a Organização Mundial de Saúde (OMS), $2002^{11}$.

A aplicação dos questionários ocorreu ou durante o $47^{\circ}$ Congresso Brasileiro de Educação Médica (COBEM), ou via e-mail ou via correio.

\section{Análise Estatística}

Adotou-se nível de significância de 5\% para testar a hipótese de nulidade por meio do programa SPSS 13.0. Foram realizadas análise descritiva, de tendência central e de variância (Teste de Mann-Whitney) para a análise dos dados.

\section{RESULTADOS}

\section{Dados Gerais do Estudo}

A taxa de adesão ao estudo foi de 32,4\% (58 das 179 escolas). A maioria dos sujeitos de pesquisa pertence ao sexo masculino $(53,4 \%)$, com média de idade de 48,6 anos, sendo oriundos 
de instituições privadas em $55,2 \%$, provenientes de diferentes regiões do Brasil.

A participação das escolas segundo as regiões do país foram: norte $8,6 \%$, nordeste $22,4 \%$, centro-oeste $5,2 \%$, sudeste $37,9 \%$ e sul $25,8 \%$

FIGURA 1- 1

A - Distribuição da amostra estudada.

B - Escolas Médicas do Brasil por região.
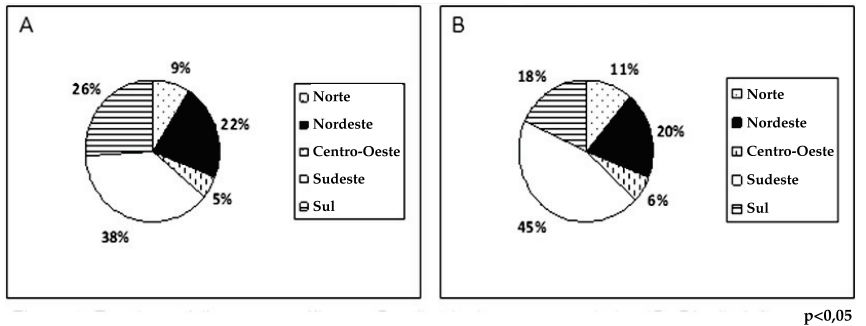

\section{Atitudes Gerais nos Cuidados no Fim da Vida}

A maioria dos coordenadores (79,3\%) considera como "muito importante" para a sua instituição como um todo, incluindo administradores e professores, que o seu aluno aprenda sobre como prover cuidados em pacientes com doença terminal, sendo que 6,9\% consideram essa questão "pouco importante". A importância para o próprio coordenador que os seus alunos aprendam sobre como prover cuidados em pacientes com doença terminal é dita como "muito importante" em 96,6\% dos casos. Eles acreditam, entretanto, que os estudantes de medicina demonstram-se "moderadamente interessados" em 48,3\% dos casos, estando "muito interessados" em 22,4\% das escolas e "pouco interessados" em 29,3\% das escolas.

Considerando os nove princípios dos cuidados paliativos propostos pela Organização Mundial de Saúde (OMS), os coordenadores das escolas médicas acreditam que nos últimos anos foi feito ou dito algo para: proporcionar alívio da dor e de outros sintomas angustiantes em $86,2 \%$ das escolas; encarar a vida e a morte como um processo normal em $73,2 \%$; não adiar nem acelerar o processo de morrer em 78,9\%; integrar os aspectos psicológicos e espirituais da assistência ao paciente em 71,9\%; oferecer sistema de apoio para ajudar os pacientes a viver tão ativamente quanto possível até a morte em 52,6\%; oferecer sistema de apoio para ajudar a família a enfrentar a doença e até mesmo o luto em $43,9 \%$; realizar abordagem em equipe para atender as necessidades dos doentes e da família, incluindo o luto, em 51,8\%; melhorar a qualidade de vida, podendo influenciar positivamente no curso da doença em 77,6\%; aplicar os princípios dos cuidados paliativos no início do curso da doença em conjugação com outras terapias des- tinadas a prolongar a vida, como a radioterapia ou quimioterapia, incluindo as investigações necessárias para melhor compreender e gerir as angustiantes complicações clínicas em $70,2 \%$ das escolas médicas estudadas.

\section{Cuidados no Fim da Vida da Vida no Currículo das Escolas Médicas}

As escolas médicas do país abordam os cuidados no fim da vida nos currículos médicos na forma de disciplina ou eletivo com enfoque primário, em 35,1\%, sendo obrigatório em $33,9 \%$ do total de escolas estudadas. Com enfoque secundário, a abordagem ocorre em $72,4 \%$, sendo compulsório em $65,5 \%$ das escolas estudadas.

Houve diferença entre a forma de fomento da instituição, pública ou privada, e a existência de disciplina com foco secundário no ensino em terminalidade da vida, sendo que este ocorre mais em instituições públicas ( $p=0,025)$.

A tabela 1 aponta para características curriculares quanto ao ensino em cuidados no fim da vida nas escolas estudadas.

TABELA 1

Características curriculares do ensino dos cuidados no fim da vida conforme a prioridade, tempo, infra-estrutura e departamento responsável pelo ensino segundo os coordenadores do curso médico.

Prioridade dada ao ensino dos cuidados no fim da vida Grande

Moderada

Pequena

Não existe

Tempo disponível para o ensino dos cuidados no fim da vida** Suficiente

Insuficiente

Excessivo

Infra-estrutura da escola médica para os cuidados dos cuidados no

fim da vida***

Adequada

Pequena adequação $\quad 21,4$

Inadequada

Departamento responsável pelo ensino dos cuidados paliativos**** Adequado

oderada adequação

Pequena adequação

Inadequado/Não existe

*Parâmetro avaliado em uma escala subjetiva e progressiva através de questão direta tendo por resposta "não existe", "pequena", "moderada" e "grande".

**Parâmetro avaliado em uma escala subjetiva e progressiva através de questão direta, tendo por resposta "insuficiente", "suficiente" e "excessivo".

***Parâmetro avaliado em uma escala subjetiva e progressiva através de questão direta, tendo por resposta "inadequada", "pequena adequação", "moderada adequação" e "adequada".

****Parâmetro avaliado em uma escala subjetiva e progressiva através de questão direta, tendo por resposta "inadequado", "mnão existe", "pequena adequação", "moderada adequação" e "adequado". 


\section{Atitudes Frente às Mudanças Curriculares}

A maioria das escolas participantes sofreu alguma reforma curricular nos últimos cinco anos (73,7\%) e em 53,8\% destas o comitê responsável pela mudança curricular não discutiu a questão do ensino dos cuidados no fim da vida dentro do novo currículo.

As percepções de atitudes que possam influenciar as mudanças curriculares no ensino dos cuidados no fim da vida na visão dos coordenadores de curso nas escolas médicas entrevistadas estão descritas na tabela 2.

\section{TABELA 2}

Percepções dos coordenaores das escolas médicas sobre as atitudes que possam modificar o ensino dos cuidados no fim da vida

\begin{tabular}{lr}
\hline & $\%$ \\
\hline $\begin{array}{l}\text { Inserção de um curso curricular obrigatório com foco primário em } \\
\text { cuidados no fim da vida - apoio dado pelos professores* }\end{array}$ & \\
$\quad$ Muito apoio & 22,8 \\
Moderado apoio & 45,6 \\
Não apóiam & 1,8 \\
Nunca foi avaliado & 29,8
\end{tabular}

Inserção de uma disciplina com foco primário em cuidados no fim da vida - apoio dado pelo coordenador*

Muito apoio

Moderado apoio

Não apóiam

Nunca foi avaliado

Frequência com que ocorre a integração do ensino em cuidados no fim da vida durante as aulas ministradas por diferentes diciplinas**

Sempre

Com muita frequência

Ocasionalmente

Nunca

Apoio dado pela instituição para a integração do ensino dos cuidados no fim da vida junto às disciplinas já existentes ${ }^{* * *}$

Muito apoio

Moderado apoio

Moderada falta de apoio

Não apoia

Apoio dado por você para a integração do ensino dos cuidados no

fim da vida junto às disciplinas já existentes*

Muito apoio

Moderado apoio

Moderada falta de apoio

Não apoia

*Parâmetro avaliado em uma escala subjetiva e progressiva através de questão direta, tendo por resposta "não apóia", "moderado apoio", "muito apoio". Caso nunca se tenha pensado na questão, considerar como "nunca foi avaliado".

**Parâmetro avaliado em uma escala subjetiva e progressiva através de questão direta tendo por resposta "nunca", "ocasionalmente", "com muita frequência" e "sempre".

***Parâmetro avaliado em uma escala subjetiva e progressiva através de questão direta, tendo por resposta "não apoia", "moderada falta de apoio", "moderado apoio" e "muito apoio".

\section{Barreiras no Ensino em Cuidados no Fim da Vida}

As barreiras relacionadas à falta de apoio da instituição, à falta de corpo docente especializado e à falta de material didático são maiores para as escolas públicas quando comparadas às instituições privadas ( $p=0,04 ; p=0,02$ e $p=0,04$ respectivamente).

TABELA 3

Barreiras no ensino em terminalidade da vida nas escolas médicas segundo a opinião dos coordenadores dos cursos de medicina

\begin{tabular}{|c|c|c|c|c|}
\hline Barreira* & Grande & Moderada & Pequena & Inexistente \\
\hline Falta de interesse da instituição & $10,3 \%$ & $25,9 \%$ & $25,9 \%$ & $37,9 \%$ \\
\hline $\begin{array}{l}\text { Falta de corpo docente } \\
\text { especializado }\end{array}$ & $25,9 \%$ & $39,7 \%$ & $15,5 \%$ & $19,0 \%$ \\
\hline $\begin{array}{l}\text { Falta de departamento } \\
\text { universitário }\end{array}$ & $14,3 \%$ & $19,6 \%$ & $28,6 \%$ & $37,5 \%$ \\
\hline Falta de verbas & $15,5 \%$ & $17,2 \%$ & $46,6 \%$ & $20,7 \%$ \\
\hline Falta de tempo & $10,5 \%$ & $33,3 \%$ & $36,8 \%$ & $19,3 \%$ \\
\hline Falta de material didático & $10,5 \%$ & $31,6 \%$ & $36,8 \%$ & $21,1 \%$ \\
\hline $\begin{array}{l}\text { Falta de um serviço clínico de } \\
\text { cuidados paliativos }\end{array}$ & $22,4 \%$ & $27,6 \%$ & $27,6 \%$ & $22,4 \%$ \\
\hline
\end{tabular}

*Parâmetro avaliado em uma escala subjetiva e progressiva através da queda direta, tendo por resposta "inexistente", "pequena", "moderada" e "grande".

\section{DISCUSSÃO}

Em 2002, a Organização Mundial de Saúde ampliou o conceito de cuidados paliativos como "a abordagem que promove qualidade de vida de pacientes e seus familiares diante de doenças que ameaçam a continuidade da vida, através de prevenção e alívio do sofrimento. Requer a identificação precoce, avaliação e tratamento impecável da dor e outros problemas de natureza física, psicossocial e espiritual." A partir deste conceito foram estabelecidos nove princípios que o norteiam ${ }^{11}$. Em relação ao ensino desses princípios, no presente estudo, os que mereceram menor atenção foram: "realizar abordagem em equipe para atender as necessidades dos doentes e suas famílias, incluindo o luto" e "oferecer sistema de apoio para ajudar os pacientes a viver tão ativamente quanto possível até a morte". O luto, como Freud observou, é extremamente doloroso, e a resposta intuitiva de muitas pessoas enlutadas é tentar minimizá-lo e evitá-lo por completo. Amigos e parentes freqüentemente cooperam com essa evitação ${ }^{12}$. Encorajar e apoiar o luto deve fazer parte da abordagem ao paciente em cuidados paliativos, sendo este o papel da equipe multidisciplinar, incluindo o médico ${ }^{11}$. Estudo anterior ${ }^{13}$ identificou que a exposição do estudante a situações hipotéticas de morte faz com que o mesmo desperte a necessidade de desenvolver estratégias para enfrentar positivamente o processo de morrer, o 
que justificaria, para o autor, a introdução e desenvolvimento de mais elementos curriculares no sentido de motivar o aluno para essa problemática. Estudantes de medicina do Rio Grande do Sul ${ }^{14}$ foram avaliados quanto aos seus sentimentos ao se depararem com o fenômeno da morte, o significado dessa experiência e suas implicações no processo de ensino-aprendizagem. Observou-se que os sentimentos e implicações no desenvolvimento pessoal e profissional do estudante são primordiais e inegáveis, sugerindo proposta psicopedagógica para a educação neste sentido ${ }^{14}$.

Nos EUA, na metade da década de 70, seis por cento das escolas de medicina ofereciam disciplina sobre a morte e o morrer $^{15}$. Já na década de 90,30\% das escolas médicas ofereciam disciplina e/ou curso dedicado aos cuidados no fim da vida, sendo que praticamente todos os programas incluíam algum aspecto desse tópico como parte de alguma disciplina ${ }^{16}$. Evidenciou-se, sobretudo na década passada, maior interesse nessa área, por conta da criação de fundo público e privado desenvolvido para a educação, cuidados clínicos e a formação de profissionais na área de cuidados no fim da vida naquele país ${ }^{17}$. Segundo a Organização Pan Americana de Saúde, nos últimos anos os cuidados paliativos estão emergindo com grande interesse na saúde pública, tanto em países desenvolvidos como naqueles em desenvolvimento ${ }^{18}$. No Brasil, com a aprovação recente da medicina paliativa como área de atuação médica ${ }^{6}$, os recursos destinados a esse campo médico tendem a aumentar, favorecendo, dessa maneira, melhoria dos indicadores encontrados no presente estudo.

Os atuais coordenadores das escolas médicas brasileiras expressam visões muito positivas sobre a importância do ensino médico em cuidados no fim da vida, entretanto ainda é dada pouca prioridade ao seu ensino. Corrobora com esta afirmativa artigo nacional que conclui que, durante o curso de medicina, pouca ênfase é dada ao estudo da morte e dos cuidados no fim da vida ${ }^{19}$. Para a maioria dos coordenadores das escolas médicas, é de grande importância tanto para a instituição, como para o coordenador, que se ensine sobre como prover os cuidados no fim da vida, não havendo diferença entre escolas públicas e privadas $(p=0,319)$. Essas observações corroboram resultados de Sullivan et al. ${ }^{10}$, embora o presente estudo conte com percentuais maiores na crença da importância no aprendizado em cuidados paliativos. Em ambos os estudos, os coordenadores acreditam que o aluno não tem grande interesse em aprender sobre como prover os cuidados no fim da vida. Tal dado merece estudo prospectivo avaliando o real interesse dos alunos com relação ao tema, com perspectiva mais realista baseada em dados coletados. Entretanto, do que se tenha notícia, este foco ainda não foi analisado no Brasil.
Assim, surge a seguinte questão: por que há uma grande discrepância entre a importância dada pelos coordenadores ao tema e a escassa visibilidade do mesmo no cenário acadêmico?

Várias são as potenciais barreiras detectadas à prática dos cuidados paliativos. As maiores entre elas, determinadas nos moldes do presente estudo, são a falta de corpo docente especializado em cuidados no fim da vida, de serviços clínicos em cuidados paliativos e de tempo alocado para o seu ensino, corroborando dados de estudo anterior ${ }^{10}$. O tempo alocado para o ensino em cuidados no fim da vida é, para a maioria das escolas nacionais, insuficiente, entretanto, o maior impasse parece estar relacionado com a falta de corpo docente especializado para esse ensino. Nos EUA, os serviços em medicina paliativa estão melhor difundidos, o que parece facilitar a realidade do ensino nas escolas médicas ${ }^{10}$. Com o reconhecimento da medicina paliativa como área de atuação médica, espera-se melhoria dos serviços especializados e dos profissionais da área, muito embora estudos realizados em alguns países nos quais a medicina paliativa é reconhecida há tempos como área de atuação médica, reafirmem a questão da falta de corpo docente qualificado ${ }^{20,21}$

Becker et $a l^{22}$ demonstraram a necessidade da educação em medicina paliativa, uma vez que determinaram que $70 \%$ dos sujeitos de sua pesquisa não consideravam ter preparo suficiente para o manejo dos cuidados no fim da vida. Na formação médica atual, as escolas médicas deveriam contemplar a questão do ensino em cuidados no fim da vida em seus currículos de maneira a direcionar os futuros profissionais para o seu dia-a-dia e propiciar a fundamentação para a formação de generalistas com habilidades paliativistas. Revisão bibliográfica do Reino Unido demonstra que a maior preocupação dos coordenadores dos cursos médicos é promover a conscientização da medicina paliativa como especialidade aplicável a seus futuros pacientes ${ }^{23}$. A existência de profissional especialista em medicina paliativa como eixo integrador, mas não exclusivamente determinante desse ensino, parece necessária no contexto do ensino atual. Além disso, considerando que a maioria das escolas possui infraestrutura adequada ao ensino dos cuidados paliativos, segundo os dados coletados no presente estudo, torna-se necessária a reflexão sobre o motivo pela qual não ocorre o favorecimento do processo de ensino-aprendizagem dos cuidados ao fim da vida. A mudança neste cenário poderia favorecer o resgate do cuidado com o paciente, muitas vezes esquecido na medicina de hoje, assim como o desenvolvimento de futuros serviços em medicina paliativa e, portanto, contribuiria para a diminuição de uma das barreiras fundamentais ao seu desenvolvimento, o pequeno número de profissionais qualificados. 
Na maioria das escolas participantes, os coordenadores consideraram que o departamento responsável pelo ensino em cuidados paliativos é inadequado ou mesmo inexistente, embora a infraestrutura permitisse sua existência, fato que pode ser reflexo tanto da forma de estruturação e administração vigente, quanto da pequena atenção voltada a esse ensino. A inexistência de departamento com este foco específico, entretanto, não parece ser barreira ao seu ensino. Estudo de Sullivan et a $l^{10}$ também considera essa variável como pequena barreira, embora não discorra sobre a adequação do departamento universitário responsável.

Outras barreiras, consideradas menores pelos coordenadores das escolas médicas analisadas, foram falta de apoio da instituição, falta de verbas e falta de material didático, achados estes similares ao estudo norte-americano ${ }^{10}$, à exceção da falta de verbas que, diferentemente do Brasil, constitui-se na terceira maior barreira. A falta de recursos foi considerada importante barreira tanto para as escolas públicas quando comparadas às instituições privadas $(p=0,254)$. Estes resultados, entretanto, não parecem ser surpreendentes já que, historicamente, o movimento hospice nunca teve grande auxílio dos sistemas de saúde tradicionais ${ }^{24}$.

O estudo demonstra que "muito apoio" é dado pelo coordenador frente à integração do ensino em cuidados no fim da vida dentro de outras disciplinas em $56,9 \%$ dos casos. Entretanto, eles acreditam que com pouca freqüência essa integração ocorra. As escolas médicas do Brasil, a partir da Reforma Universitária de 1968, passaram a adotar oficialmente o modelo norte americano de ensino. $\mathrm{O}$ ensino fica dividido em dois ciclos, um de disciplinas básicas e outro de disciplinas profissionalizantes. Os currículos de graduação, dessa forma, apresentam estrutura que tem permitido acrescentar conhecimentos, mostrando número crescente de disciplina. O que significou e, foi reconhecido inicialmente, como progresso passa a constituir-se de problema em determinadas situações, devido a pouca interação entre as disciplinas, mesmo fazendo parte do mesmo departamento e estando ligadas à formação de mesmo perfil profissional ${ }^{25}$. O presente estudo reflete a influência deste modelo Flexneriano de educação, posto que verificou que 59,6\% dos coordenadores dizem dar "muito apoio" à criação de disciplina com foco primário em terminalidade da vida. O paradigma da integralidade busca contrapor e equilibrar o paradigma flexneriano e tem como objetivo-imagem a ser alcançada, no que se refere aos programas de graduação do profissional da saúde, formação mais contextualizada, que leve em conta as dimensões sociais, econômicas e culturais da vida da população, instrumentalizando os profissionais para enfrentar os problemas do processo saúde-doença da população ${ }^{26}$. Esse princípio operativo a nortear o movimento de mudança, deveria estar sustentado na integração curricular, em modelos pedagógicos mais interativos, na adoção de metodologias de ensino-aprendizagem centradas no estudante como sujeito da aprendizagem e no professor como facilitador da construção de conhecimento ${ }^{27}$. Duas estratégias podem ser utilizadas para o ensino do tema: contínua e com foco primário ou não contínua, não obrigatória e abordada em disciplinas ligadas ao tema da morte e dor ${ }^{4,5}$. O presente estudo demonstrou que disciplina ou eletivo obrigatório com foco primário está presente na minoria das escolas estudadas. As taxas percentuais aumentam ao se tratar de disciplina ou eletivo com foco secundário em terminalidade de vida. Esses dados podem ser reflexo da tendência mundial da integração do ensino em cuidados paliativos dentro de disciplinas curriculares pré-existentes, entretanto, a maioria dos participantes deste estudo acredita que essa integração do ensino dos cuidados paliativos junto a outras disciplinas pré-existentes é feita em freqüência pequena. De forma interessante, este estudo demonstrou que o ensino dos cuidados no fim da vida, com seus tópicos ministrados sob foco secundário ocorre mais em instituições públicas $(p=0,02)$ quando comparado às instituições privadas, merecendo estudos dirigidos quanto a esse achado. Pesquisadores do assunto ${ }^{28}$ concluíram que currículo médico sem curso obrigatório em medicina paliativa aumenta a confiança em algumas competências exigidas neste âmbito, mas os resultados globais no final da formação são ruins. Estes autores sugerem que estas conclusões possam servir como base para avaliar o efeito dos cursos obrigatórios em cuidados paliativos. O mesmo estudo, entretanto, não observou mudanças em relação à comunicação de más notícias, integração dos aspectos espirituais do doente e acompanhamento do paciente em processo de morrer em escolas que não possuíam curso obrigatório com foco primário em medicina paliativa ${ }^{28}$. Recentemente, pode-se verificar que a presença de disciplina obrigatória em cuidados no fim da vida nas escolas médicas promove maior efetividade na aprendizagem dos princípios em cuidados paliativos quando comparadas àquelas onde não há obrigatoriedade da disciplina ${ }^{29}$. No entanto, mais importante do que agregar conhecimentos em medicina paliativa é a mudança de atitude na prática com o paciente com doença terminal. Alguns estudos demonstraram mudança de atitude em relação ao manejo de pacientes com doença terminal quando se tem treinamento efetivo em medicina paliativa ${ }^{30,31,32,33}$.

Na visão dos coordenadores de cursos, a instituição demonstra apoiar a integração no processo de ensino, visto que a maioria acredita que é dado apoio, muito ou moderado, a esta ação que, entretanto, não acontece. Embora, na prática, ainda 
ocorra mais ações baseadas no modelo Flexneriano, começa-se a se pensar na integralidade com a importância que ela merece na educação médica. Estudos internacionais, corroboram com o modelo da integralidade no ensino dos cuidados paliativos $^{10,21}$. Metanálise ${ }^{21}$ sobre o ensino e aprendizagem em cuidados paliativos na graduação em medicina, demonstrou falta de consistência sobre o que deve ser ensinado em cuidados paliativos, tendo sido considerado como ensino fragmentado, com grande dificuldade no recrutamento de professores especializados e ausência de currículo formal na maioria das vezes. Alertou ainda para o fato do ensino estar mais focado na aquisição de conhecimentos do que na mudança de atitude do aluno de graduação ${ }^{21}$. Análise sobre os fatores que estimulam ou dificultam a incorporação do ensino dos cuidados paliativos na graduação médica no Reino Unido detectou que, além daqueles fatores comuns a todas as especialidades médicas, há fatores adicionais como a necessidade de chefe de departamento, estrutura curricular, características do paciente e exposição, presença de profissionais e serviços locais especializados, apoio da universidade e influência dos alunos ${ }^{20}$. Concluiu que a incorporação dos cuidados paliativos no currículo médico da graduação envolve complexo processo individual, institucional, curricular e do paciente ${ }^{20}$.

Como tentativa de padronizar a forma do ensino dos cuidados paliativos, a European Association of Palliative Care ${ }^{34} \mathrm{pu}-$ blicou documento intitulado "Curriculum in Palliative Care for Undergraduate Medical Education" a qual traz recomendações para os países europeus para montar estratégias quanto à implantação do ensino em cuidados no fim da vida. As únicas publicações de caráter institucional que se tem notícia no Brasil tem origem no Conselho Regional de Medicina do Estado de São Paulo (CREMESP) ${ }^{4}$, além de revisão bibliográfica ${ }^{19}$ que conta com algumas sugestões.

Muito embora o estudo apresente amostra pequena devido à adesão obtida, a distribuição entre os participantes é similar à distribuição das escolas médicas do país por região (figura 1). Pode-se afirmar que a pequena adesão ao estudo não o limita, já que a amostra tornou-se representativa da população que lhe deu origem. A falta de participação no presente estudo pode refletir a inexistência do ensino dos cuidados paliativos nessas escolas, além de justificativas de cunho psicológico, metodológico e operacional. Sullivan et al ${ }^{10}$, com objetivos e metodologias similares, contou com a participação de 52 (82\%) escolas médicas norte-americanas, enquanto outra pesquisa norte-americana ${ }^{35}$, obteve participação de $23,4 \%$ dos coordenadores dos cursos médicos, índice inferior ao apresentado no presente estudo. Infelizmente não contamos, em nosso país, com base de dados similar ao norte-americano, o que facilitaria a consulta entre os pares e acesso indiscriminado ao conteúdo ministrado nas escolas médicas nacionais. Esta lacuna das entidades que controlam o cenário educacional médico dificulta a possível obtenção de dados. Muito embora a ausência de banco de dados nacional, para o presente estudo não tivesse representado grande impacto na casuística final, tal realidade cria dificuldades quanto à obtenção de dados fidedignos que visem à melhoria do ensino médico nacional.

Considerando os dados analisados, o ensino em cuidados no fim da vida no Brasil parece manter-se deficitário. Espera-se que essa análise estimule reflexões quanto a este importante tema. Discussão nacional sobre o tema, em especial das escolas médicas que já disponham deste serviço e, a partir de então, documento que padronize como devem ser ensinados os cuidados paliativos, à exemplo da Europa, são necessários para atender a realidade atual do Brasil. Os resultados encontrados revelam as potenciais mudanças para melhoria no ensino em cuidados no fim da vida, os quais devem ser analisados pelas escolas médicas considerando sua realidade local, de forma a identificar tanto falhas como oportunidades de melhorias nesse ensino. Para isto, algumas limitações do estudo devem ser consideradas. O primeiro ponto a ser destacado é que o instrumento de pesquisa foi utilizado em estudo prévio norte-americano ${ }^{10} \mathrm{e}$ adaptado para a realidade brasileira, entretanto, ele não é instrumento validado na literatura e possui limitações estruturais para abranger as diferentes tipologias de escolas médicas existentes no país. Segundo ponto é que há subjetividade quanto aos resultados encontrados, a qual se deve às informações não baseadas nos tópicos curriculares abordados, por meio planos de disciplinas das escolas médicas, mas sim ao ponto de vista dos coordenadores em relação ao ensino em cuidados no fim da vida.

\section{CONCLUSÃO}

As atitudes e práticas abordadas no currículo das escolas médicas estudadas, conforme reportadas pelos coordenadores de curso e, dentro das limitações do estudo, sugerem que o ensino em cuidados no fim da vida no Brasil possui limitações, necessitando de ampla discussão. Assim, as evidências sugerem que mais esforços devem ser direcionados para a melhoria da realidade deste importante tópico na formação médica, não exclusivamente por parte escolas médicas, mas de órgãos de Educação e Saúde do Brasil.

\section{REFERÊNCIAS}

1. Doyle D, Hanks GW, MacDonald N. Oxford textbook of palliative medicine. Oxford (UK): Oxford University Press;1998. 
2. Wright M, Wood J, Lynch T, Clark D. Mapping Levels of Palliative Care Development: A global view. J Pain Symptom Manage. 2008;35(5):469-85.

3. Support Principal Investigators. A controlled trial to improve care for seriously ill hospitalized pacients. Study to Undestand Prognoses and Preferences for Outcomes and Risks of Treatment (SUPPORT). The SUPPORT Principal Investigators. JAMA 1995;274(20):1591-8.

4. Oliveira RA, coord. Cuidado Paliativo. São Paulo: Conselho Regional de Medicina do Estado de São Paulo; 2008.

5. Brasil. Ministério da Educação. Conselho Nacional de Educação. Câmara de Educação Superior. Resolução CNE/ CES no 4 de 7 de novembro de 2001. Institui diretrizes curriculares nacionais do curso de graduação em Medicina. Diário Oficial da União, 9 nov. 2001; Seção 1, p.38.

6. Conselho Regional de Medicina do Estado de São Paulo. Medicina Paliativa é oficializada como área de atuação diz Jornal do CREMESP [online]. 2010 [acesso em 20 dez. 2010]. Disponível em www.cremesp.org.br.

7. Conselho Federal de Medicina. Código de Ética Médica. Resolução CFM no 1931, de 17 de setembro de 2009. Aprova o Código de Ética Médica. Diário Oficial da União, 24 set. 2009. Seção I, p. 90-2.

8. Instituto Nacional de Estudos e Pesquisas Anísio Teixeira [homepage]. Brasília: Ministério da Educação [Acesso em 20 fev. 2011]. Disponível em: http:/ /www.inep.gov.br/superior/censosuperior/sinopse/default.asp

9. Associação Brasileira de Educação Médica [homepage]. Rio de Janeiro: ABEM [acesso em 15 mar. 2010]. Disponível em: http:/ / www.abem-educmed.org.br/

10. Sullivan AM, Warren AG, Lakoma MD, Liaw KR, Hwang D, Block SD. End-of-life care in the Curriculum: A National Study of Medical Education Deans. Acad Med. 2004;79(8):760-8.

11. World Health Organization. Pain Relief and Palliative Care. 2a ed. Geneva: WHO; 2002.

12. Kahn M. Freud básico: pensamentos pasicanalíticos para o século XXI. Rio de Janeiro: Civilização Brasileira; 2003.

13. Williams CM, Wilson CC, Olsen CH. Dying, death and medical education: student voices. J Palliative Med. 2005;8(2):372-81

14. Carpena LA. Death versus feelings: a reality in the world of medical students. Rev Gaucha Enferm. 2000;21(1):100-22.

15. Dickinson GE. Death education in U.S. medical schools: 1975-1980. Med Educ.1981;2(56):111-14.

16. Barzansky B, Veloski JJ, Miller R, Jonas HS. Education in end-of-life care during medical school and residency training. Academic Medicine 1999; 10(74):102-104.
17. 17.Kass-Bartelmes BL, Hughes R, Rutherford MK. Advance care planning: preferences for care at the end of life. Rockville (MD): Agency for Healthcare Research and Quality. Research in Action. 2003;12.

18. Lima L, Bruera E. The Pan American Health Organization: its structure and role in the development of a palliative care program for Latin American and the Caribbean. J Pain Symptom Manage. 2000;20(6):440-8.

19. Lago PM, Lopes MH. Cuidados com o final da vida: como abordar este difícil tema? Sci Med. 2005; 1(15):47-51.

20. Gibbins J, McCoubrie R, Maher J, Forbes K. Incorporating palliative care into undergraduate curricula: lessons for curriculum development. Med.Educ. 2009;43(8):776-83.

21. Lloyd-Williams M, MacLeod RD. A systematic review of teaching and learning in palliative care within the medical undergraduate curriculum. Med Teach. 2004;26(8):683-90.

22. Becker G, Momm F, Gigl A, Wagner B, Baumgartner J. Competency and educational needs in palliative care. Wien Klin Wochenschr. 2007;119(3-4):112-6.

23. Gibbins J, McCoubrie R, Maher J, Wee B, Forbes K. Recognizing that it is part and parcel of what they do: teaching palliative care to medical students in the UK. J Palliat Med. 2010;24(3):299-305.

24. St. Christopher's Hospice. Annual review 2007/2008: Responding to Challenge and Change. [Acesso em 01 jan. 2012] London: St Christopher's Hospice; 2008. Disponível em: www.helpthehospices.org.uk

25. Lampert JB. Tendências de mudanças na formação médica no Brasil: tipologia das escolas. São Paulo: Hucite; 2009.

26. 26.Campos FE, Ferreira JR, Feuerwerker L, Sena RR, Campos JJB, Cordeiro H, Cordini Júnior L. Caminhos para aproximar a formação de profissionais de saúde das necessidades da atenção básica. Rev Bras Educ Med. 2001;25(2):53-9.

27. 27.Feuerwerker LCM, Sena RA. Educação dos profissionais de saúde na América Latina: teoria e prática de um movimento de mudança. São Paulo: Hucitec; 1999.

28. 28. Ostgathe C, Voltz R, Nauck F, Klaschik E. Undergraduate training in palliative medicine in Germany: what effect does a curriculum without compulsory palliative care have on medical students' knowledge, skills and attitudes? J Palliat Med. 2007;21(2):155-6.

29. Toledo AP, Martinello LZ, Priolli DG. Disciplina com foco primário em terminalidade da vida: sua obrigatoriedade é válida? In: Anais do IV Congresso Internacional de Cuidados Paliativos; 2010 Out. 6-9; Instituto de Ensino e Pesquisa do Hospital Sírio Libanês São Paulo, Brasil. São Paulo, SP: ANCP, c2010. 131p. 
30. 30.Gruber PC, Gomersall CD, Joynt GM, Lee A, Tang PY, Young AS, Yu NY, Yu OT. Changes in medical students' attitudes towards end-of-life decisions across different years of medical training. J Gen Intern Med. 2008;23(10):1608-14.

31. 31.Fraser HC, Kutner JS, Pfeifer MP. Senior medical students' perceptions of the adequacy of education on end-of-life issues. J Palliat Med. 2001 Fall;4(3):337-43.

32. Tsai SS, Hu WY, Chang HH, Chang SC, Chen CY, Chiu TY. Effects of a multimodule curriculum of palliative care on medical students. J Formos Med Assoc. 2008;107(4):326-33.

33. 33.Mason SR, Ellershaw JE. Preparing for palliative medicine; evaluation of an education programme for fourth year medical undergraduates. J Palliat Med. 2008;22(6):687-92.

34. European Association for Palliative Care Onlus. Curriculum in Palliative Care for Undergraduate Medical Education. Recommendations of the EAPC. [acesso em 20 set. 2010] Milão: EAPC; 2007. Disponível em: www.eapcnet.org
35. Van Aalst-Cohen ES, Riggs R, Byock IR. Palliative care in medical school curricula: A Survey of United States Medical School. J Palliat Med. 2008;11(9):1200-2.

\section{CONTRIBUIÇÃO DOS AUTORES}

As autoras contribuíram na concepção e desenho do estudo, aquisição, análise e interpretação de dados, elaboração da versão inicial do artigo e sua revisão crítica.

\section{CONFLITO DE INTERESSES}

Declarou não haver.

\section{ENDEREÇO PARA CORRESPONDÊNCIA}

Denise Gonçalves Priolli

Rua São Vicente, 614

Jardim Paulista - Atibaia

CEP: $12947-390$ SP

E-mail: depriolli@terra.com.br 